\title{
Metabonomics adds a new dimension to fragile $X$ syndrome
}

Inge Heulens ${ }^{\dagger}$, Sien Braat ${ }^{\dagger}$ and R Frank Kooy*

\begin{abstract}
Fragile $X$ syndrome is the most common cause of inherited intellectual disability, but the underlying pathophysiology is complex and effective treatments are lacking. In a recent study of fragile $X$ mental retardation 1 (Fmr1) knockout mice, the metabolic profile of the fragile $X$ brain was determined using proton high-resolution magic angle spinning nuclear magnetic resonance spectroscopy. This analysis revealed deficiencies in four metabolic categories: neurotransmission, osmoregulation, energy metabolism and oxidative stress response. Abnormalities in the metabolic phenotype were linked to the fragile $X$ mental retardation protein using an integrated metabolome and interactome mapping approach, allowing a global picture of the disorder to emerge.

Keywords Fragile $X$ syndrome, metabonomics, knockout mouse, FMR1, FMRP, GABA, glutamate, gene networks
\end{abstract}

\section{The fragile $X$ protein has many interactions}

Since the discovery of the fragile $X$ mental retardation 1 (FMR1) gene in 1991, our knowledge of the molecular mechanisms underlying fragile $\mathrm{X}$ syndrome (FXS) has increased tremendously [1]. The disorder is caused by dynamic mutation of a single trinucleotide sequence, CGG, on the $\mathrm{X}$ chromosome. In patients, CGG repeat expansion and subsequent methylation silences the FMR1 gene, preventing transcription of the fragile $\mathrm{X}$ mental retardation protein (FMRP).

FMRP is an RNA binding protein involved in mRNA transport and stability and also in local protein translation. The protein is estimated to interact with $4 \%$ of all

These authors contributed equally

*Correspondence: Frank.Kooy@ua.ac.be

Department of Medical Genetics, University of Antwerp, Prins Boudewijnlaan 43, 2650 Edegem, Belgium brain mRNAs and with an unknown number of proteins in the mammalian brain. Absence of FMRP leads to perturbation of the normal cellular function of many mRNAs and proteins. The resulting fragile $\mathrm{X}$ phenotype is characterized by intellectual disability, specific physical abnormalities, behavioral problems and epileptic seizures. Currently, there is no specific treatment for the disorder. Elucidating the pathophysiology of FXS is therefore not only important for the identification of potential therapeutic targets, but could also provide further insights into the general mechanisms of cognition and behavior.

Various techniques have been used to determine the mRNA targets of FMRP, including co-immunoprecipitation followed by microarray analysis, antibody-positioned RNA amplification (APRA) and, most recently, highthroughput sequencing of RNAs isolated by crosslinking immunoprecipitation (HITS-CLIP) [2]. Each study identified a large number of mRNA targets, encoding proteins involved in synaptic signaling pathways, such as glutamate and $\gamma$-aminobutyric acid (GABA), in addition to proteins involved in many other intracellular pathways. Proteomic analysis of murine hippocampal synapses revealed abnormalities due to loss of translational regulation of FMRP [3]. Altered expression profiles were found predominantly for proteins in specific functional groups, such as synaptic structure and cytoskeleton organization, neurotransmission and metabolism.

A recent study by Davidovic and colleagues [4], published in Genome Research, provides a new dimension to fragile $\mathrm{X}$ research by analyzing the brain metabolome of Fmr1-deficient mice. By combining metabonomics data with results from previously published studies and with the knowledge of signaling and metabolic pathways gathered from databases, this work significantly increases our insight into the aberrant signaling pathways in FXS.

\section{Metabonomics as a tool to study genetic disorders}

Metabonomics measures the dynamic multiparametric metabolic response of living systems to pathological stimuli or genetic modification [5]. The collection of all metabolites (the intermediate and end products of cellular processes) in a biological sample is represented by the metabolome, which can reflect the ultimate response of 


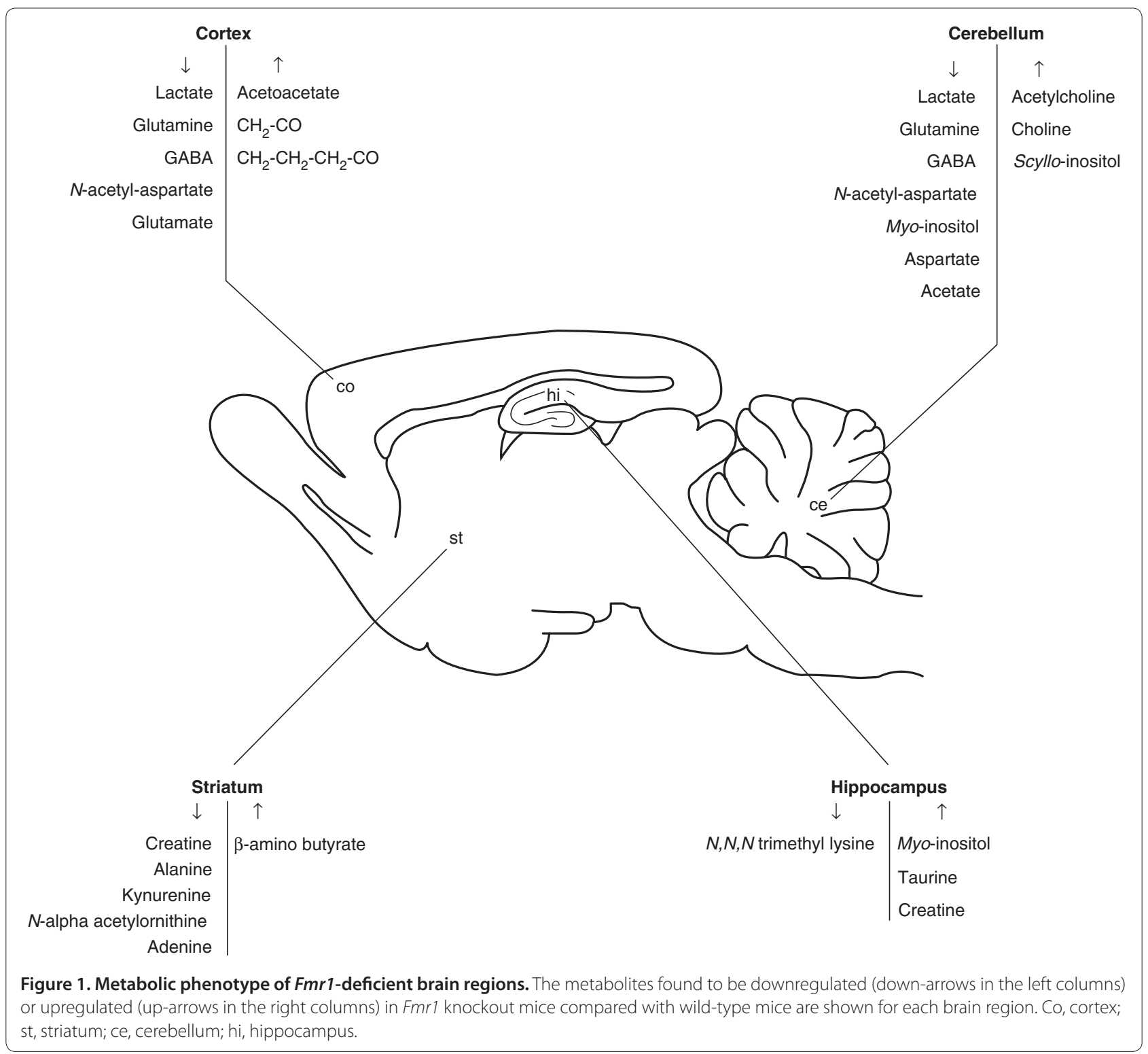

biological systems to genetic or environmental changes [6]. Metabonomics is a rapidly developing field with many potential applications in cancer and neurodegenerative disease diagnostics, monitoring of disease progression, evaluation of drug toxicity and development of therapeutics [7]. It can also be used to identify the underlying pathways involved in a disease.

Traditionally, genomics and proteomics approaches have been used to study the effect of genetic disorders. However, finding the relationship between altered gene or protein expression and the biological consequences is not always straightforward [5], especially given that the effect of a single genetic variation is often not limited to one biochemical pathway [6]. Metabonomics can help to identify the metabolites of seemingly unrelated biochemical pathways, and therefore might reveal potential new sites for therapeutic intervention. The metabolites can be detected by approaches based on mass spectrometry or nuclear magnetic resonance (NMR) spectroscopy. In their study, Davidovic et al. [4] used solid state NMR, specifically ${ }^{1} \mathrm{H}$ magic-angle-spinning NMR ( ${ }^{1} \mathrm{H}$ HR-MAS NMR) spectroscopy. This method has the advantage of being able to analyze small amounts of intact tissues, unlike other methods, which require metabolites to be in solution. The technique can thus be used even if further analysis of the tissue sample is required.

\section{Defects in key metabolic pathways}

The outcome of the metabonomic analysis showed 25 metabolites with altered concentrations in the fragile $\mathrm{X}$ 
brain (Figure 1). These 25 metabolites could be grouped into a small number of affected pathways, involved in one of four categories: neurotransmission, osmoregulation, energy metabolism and oxidative stress response. Some alterations are in line with reported anomalies in FXS, including abnormalities in the glutamatergic and the GABAergic pathways [1]. For instance, a reduced amount of GABA in the cortex and cerebellum is compatible with earlier reports of a compromised GABAergic system in the disorder. Increased glutamatergic signaling is another hallmark of the disorder [8]. It can be hypothesized that the reduced amount of free glutamate observed by Davidovic et al. [4] is a protective reaction of the organism to the overstimulation of the group I metabotropic glutamate (mGluR) receptors. There have been isolated reports that the cholinergic system, a second inhibitory system in brain, is also involved in the disorder, mainly on the basis of results from fly studies [9]. The Davidovic et al. study [4] clearly shows that several components in the cholinergic pathway are compromised in the mouse model. This adds further evidence to the hypothesis that FXS is a consequence of the disturbance of the delicate balance between excitatory and inhibitory signaling in the brain.

To integrate the abnormal metabolite concentrations with the data available from previous studies, the authors [4] developed a novel integrated metabolome and interactome mapping (iMIM) approach. This systems biology approach was considered necessary because FMRP does not interact directly with the metabolites themselves and therefore cannot directly influence their concentrations. All the 25 metabolites were linked to FMRP in the network by, on average, just over three intermediate proteins. The distance within the network represents the mechanistic link between FMRP and each metabolite. Within the interaction network, only 12 mRNAs and one protein are linked directly to FMRP. Of these, some are known interactors, including the paralogous protein FXR2P and mRNAs encoding subunits of the GABA receptor. Interestingly, a key interactor is the amyloid beta precursor protein (APP) mRNA, which is implicated in Alzheimer's disease. A previous study indicated that FMRP binds $A P P$ mRNA, but it is striking that $A P P$ is predicted to be in the center of the metabolite network, implying a pivotal role. Further studies are needed to evaluate the role of $A P P$ in FXS.

Another key interactor of FMRP emerging from this study is RhoA mRNA. RhoA is a member of the Rho GTPase family, and different members of this gene family have opposite roles in neurite outgrowth and retraction. Although it was known that the Rho GTPase Rac1, which promotes neural spine formation, was involved in the disorder, this study supports the involvement of the RhoA signaling pathway, which induces spine retraction.
In line with this, expression of Larg, encoding a protein that activates RhoA, was reported to be compromised in FXS in an earlier study [10]. These findings emphasize the involvement of Rho GTPases in the neuro-anatomical pathophysiology of FXS, more specifically in the aberrant spine morphology associated with the disease.

\section{The fragile $X$ syndrome profile overlaps with that of other neurological disorders}

This story [4] adds another layer of understanding to the complex phenotype of FXS and it contains both good and bad news for the design of drugs to treat the disorder. Although it confirms a key role of inhibitory and excitatory pathways, the abnormalities in these and other affected systems seem to be specific to particular brain regions and thus may require much more sophisticated drug treatments than currently anticipated [1]. In addition, the abnormalities were detected in very young animals and an effect of brain development on the disturbances of metabolite concentrations cannot be excluded. Further studies on mice in different age groups are necessary to clarify this issue.

The study from Davidovic and colleagues [4] highlights many similarities between the metabolome of FXS and that of other neurological disorders. Examples include the neurodevelopmental disorder Rett syndrome and the neurodegenerative disorders Batten disease and Huntington's disease, which have been analyzed by other groups using metabonomics [7]. Alterations in the balance between the excitatory (glutamate) and inhibitory (GABA) neurotransmitters, osmoregulation (myo-inositol), lipid metabolism (choline) and energy metabolism (creatine) were the most commonly observed metabolic changes across all these diseases. Thus, collectively these findings contribute to our understanding of the mechanisms underlying neurological disorders in general.

\section{Abbreviations}

APP, amyloid beta precursor protein; FMR1, fragile $X$ mental retardation 1 gene: FMRP, fragile X mental retardation protein; FXS, fragile X syndrome; GABA, $Y$-aminobutyric acid; Larg, leukemia-associated Rho guanine nucleotide exchange factor; NMR, nuclear magnetic resonance; RhoA, Ras homology protein A.

\section{Competing interests}

The authors declare that they have no competing interests.

\section{Authors' contributions}

All authors participated in writing the manuscript.

\section{Acknowledgements}

We are supported by the Agency for Innovation by Science and Technology in Flanders (IWT) and the Belgian National Fund for Scientific Research - Flanders (FWO).

Published: 28 December 2011

\section{References}

1. Rooms L, Kooy RF: Advances in understanding fragile $X$ syndrome and related disorders. Curr Opin Pediatr 2011, 23:601-606. 
2. Darnell JC, Van Driesche SJ, Zhang C, Hung KY, Mele A, Fraser CE, Stone EF, Chen C, Fak JJ, Chi SW, Licatalosi DD, Richter JD, Darnell RB: FMRP stalls ribosomal translocation on mRNAs linked to synaptic function and autism. Cell 2011, 146:247-261.

3. Klemmer P, Meredith RM, Holmgren CD, Klychnikov Ol, Stahl-Zeng J, Loos M van der Schors RC, Wortel J, de Wit H, Spijker S, Rotaru DC, Mansvelder HD, Smit AB, Li KW: Proteomics, ultrastructure and physiology of hippocampal synapses in a Fragile X Syndrome mouse model reveals pre-synaptic phenotype. J Biol Chem 2011, 286:25495-25504.

4. Davidovic L, Navratil V, Bonaccorso CM, Catania MV, Bardoni B, Dumas ME: A metabolomic and systems biology perspective on the brain of the Fragile X syndrome mouse model. Genome Res 2011, 21:2190-2202.

5. Nicholson JK, Lindon JC, Holmes E: 'Metabonomics': understanding the metabolic responses of living systems to pathophysiological stimuli via multivariate statistical analysis of biological NMR spectroscopic data. Xenobiotica 1999, 29:1181-1189.

6. Fiehn O: Metabolomics--the link between genotypes and phenotypes. Plant Mol Biol 2002, 48:155-171.
7. Holmes E, Tsang TM, Tabrizi SJ: The application of NMR-based metabonomics in neurological disorders. NeuroRx 2006, 3:358-372.

8. Bear MF, Huber KM, Warren ST: The mGluR theory of fragile X mental retardation. Trends Neurosci 2004, 27:370-377.

9. Chang S, Bray SM, Li Z, Zarnescu DC, He C, Jin P, Warren ST: Identification of small molecules rescuing fragile $X$ syndrome phenotypes in Drosophila. Nat Chem Biol 2008, 4:256-263

10. Gantois I, Vandesompele J, Speleman F, Reyniers E, D'Hooge R, Severijnen L-A, Willemsen R, Tassone F, Kooy RF: Expression profiling reveals involvement of the $\mathrm{GABA}_{A}$ receptor subunit $\delta$ in the fragile $\mathrm{X}$ syndrome. Neurobiol Dis 2006, 21:346-357.

doi:10.1186/gm296

Cite this article as: Heulens I, et al:: Metabonomics adds a new dimension to

fragile X syndrome. Genome Medicine 2011, 3:80. 